\title{
Les Trajectoires d'une Reconversion du Militantisme Associatif au Courtage en Développement : Le Cas de Timidria au Niger
}

\section{Mahaman Tidjani Alou}

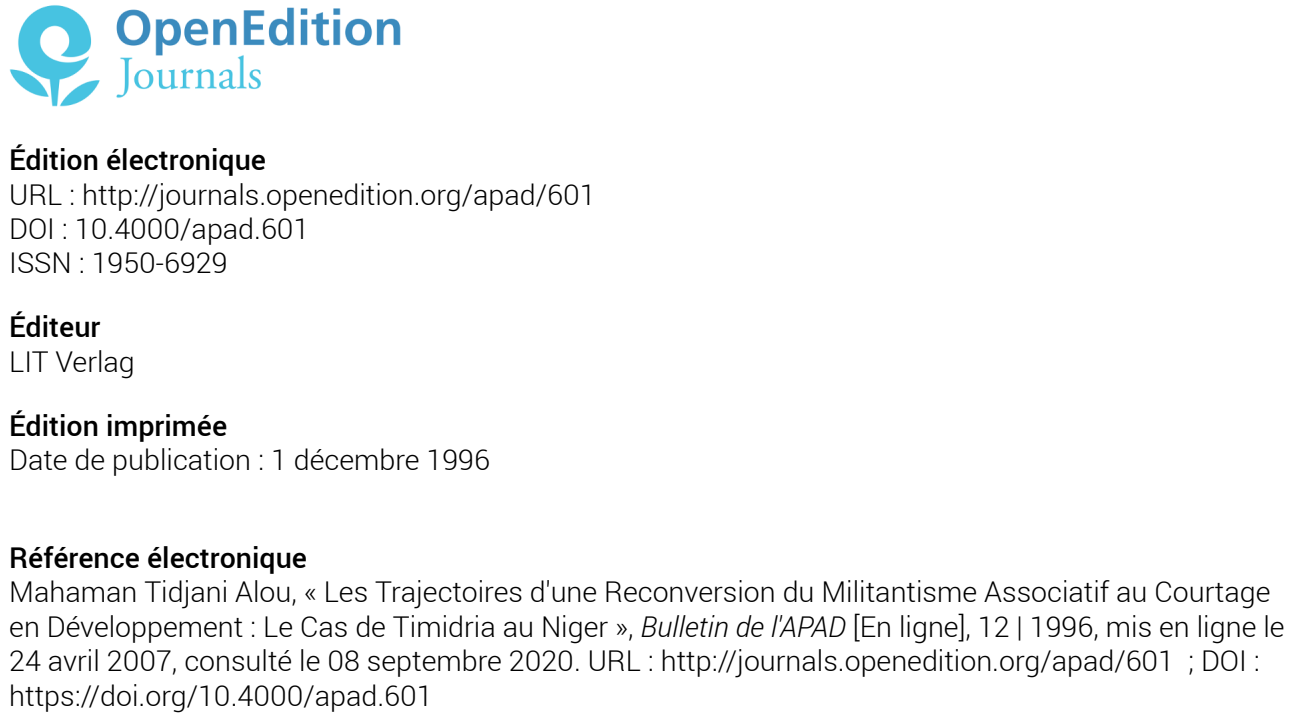

Ce document a été généré automatiquement le 8 septembre 2020

Bulletin de I'APAD 


\title{
Les Trajectoires d'une Reconversion du Militantisme Associatif au Courtage en Développement : Le Cas de Timidria au Niger
}

\author{
Mahaman Tidjani Alou
}

1 L'effervescence associative a été incontestablement un point marquant des processus de démocratisation engagés dans les Etats africains à la fin des années 1980. Mais notre objet ici n'est pas de l'étudier en tant que tel. Il s'agit surtout de l'utiliser comme espace empirique pour comprendre des acteurs fort peu étudier sous cet angle en anthropologie, les courtiers locaux du développement.

2 Par courtiers locaux de développement, il faut entendre "des acteurs sociaux implantées dans une arène locale qui servent d'intermédiaires pour drainer (vers l'espace social correspondant) des ressources extérieures relevant de ce qu'on appelle communément l'aide au développement" 1 .

3 La notion de courtier, mais à condition de l'écarter des connotations classiques qu'elle induit ${ }^{2}$, peut renvoyer à plusieurs terrains. Au fonds, la notion de courtage en développement peut servir d'outil d'analyse du rôle joué par de nombreux intervenants dans les actions de développement. Dans ce sens, il est possible d'identifier de nombreux espaces, de "surface variable", qui se spécifient tant par la nature de leurs actions que par la configuration particulière que ces actions impriment dans des champs sociaux déterminés. A un certain niveau d'analyse, un gouvernement, une structure étatique donnée, ou encore une organisation internationale comme le PNUD assure dans certains aspects de leurs actions des fonctions de courtage. Cependant, l'ambition ici n'est pas d'aborder ces grands ensembles, même si cette fonction, sous d'autres registres, a sûrement fait l'objet d'études sans que la notion de courtage ait pu être utilisée pour la qualifier. Nous voulons surtout fonder cette recherche en observant le terrain associatif constitué ces dernières années au Niger à la faveur du processus de démocratisation qui s'y est enclenché à partir de 1990. 
Timidria ${ }^{3}$. Créée le 15 mai 1991, elle est officiellement reconnue en décembre de la même année. Son siège est à Niamey.

Cette association a été peu étudiée. On trouve des éléments la concernant dans un rapport de mission élaboré par L. Barbedette et J. Ouédraogo dans le cadre d'une consultation plus large qu'ils ont effectuée au Niger sur le milieu associatif nigériens ${ }^{4}$.

On trouve aussi un article plus récent de Mme Schlechten Rauber parut dans un ouvrage collectif récent. L'auteur qui a beaucoup travaillé avec l'association dans le cadre de ses activités professionnelles voit en elle une dynamique inédite au Niger ${ }^{5}$. Cette somme, bien qu'assez faible constitue référence de départ qu'il a fallu compléter par des enquêtes de terrain.

7 Selon L. Barbedette et J. Ouédraogo, Timidria dispose d'environ 80.000 membres. Mais quand on cherche à confirmer ce nombre auprès des membres actifs du bureau, ils reconnaissent ne pas le savoir exactement car "des gens s'autoproclament Timidria sans le notifier au bureau". Néanmoins, il semble que l'association soit implantée actuellement dans 5 départements sur les 8 que compte le Niger. Son enracinement spatial accrédite l'idée d'une forte implantation dans les régions qui recensent de fortes implantations touareg ${ }^{6}$. C'est ainsi qu'on retrouve parmi les sections de l'association représentées au séminaire qu'elle a organisé à Tahoua ${ }^{7}$, des représentants d'Agadez, de Tillabéry, de Maradi, de Tahoua et de la communauté urbaine de Niamey ${ }^{8}$.

8 Il faut dire qu'au début de nos enquêtes, cette association, dans ses nombreuses activités (manifestations artistiques, sensibilisation dans des réunions) répondait difficilement aux critères définis pour identifier les courtiers locaux du développement, $\mathrm{Au}$ départ donc le terrain était loin d'être pertinent. Mais il l'est devenu progressivement, à mesure que les activités de Timidria prenaient de l'ampleur et que sa présence dans le paysage associatif était devenue palpable. Elle nous donnait donc l'occasion de poser notre problème autrement, en focalisant l'attention, non pas sur les courtiers en tant que tels, mais sur la dimension génétique du problème. Une question simple venait à l'esprit. Comment devient-on courtier ? Question simple, il est vrai, mais qui nous donnait l'occasion d'observer un processus. Il s'agissait donc d'examiner le processus qui conduit certains militants d'associations à devenir des courtiers locaux du développement. Comment intègrent-ils progressivement dans leur militantisme le courtage en développement? Comment, en s'investissant dans le courtage en développement, acquièrent-ils des ressources nouvelles, inédites, qui les confortent dans leurs activités nouvelles?

militants, militants, mêmes les plus actifs d'entre eux, ne deviennent pas nécessairement des courtiers du développement. Ceux qui le deviennent sont toujours détenteurs de ressources particulières qui les y prédisposent, mais qu'ils doivent ensuite accroître pour se maintenir sur le marché du développement. Ils doivent toujours acquérir des compétences utiles à l'efficacité de leurs actions de courtage. Par ailleurs, l'exemple de Timidria nous a permis de voir que devenir courtier n'est pas toujours le produit d'une action volontariste préméditée par les acteurs. Parfois, certains le deviennent malgré eux par leur capacité à gérer de manière continue des activités de l'association qu'ils ont la charge de conduire. L'action associative ne conduit pas toujours au courtage en développement. Et pour ceux qui deviennent courtiers, il y a toujours un temps d'apprentissage plus ou moins long et dépendant des ressources qu'ils réussissent à 
capter et de la gestion qu'ils arrivent à en faire. Le parcours est parfois semé d'embûches diverses, de nature à les retarder ou à les éliminer du chemin leur permettant de devenir courtier du développement.

10 Notre démarche nous conduira à observer que les courtiers du développement émergent dans un contexte international favorable. Sur la base d'un militantisme associatif, rendu possible par les politiques de démocratisation engagées dans les pays africains, et constituant leur ressource initial, les courtiers développent des actions qui, à mesure qu'elles prennent de l'ampleur, développent chez eux des ressources nouvelles qui les positionnent progressivement en tant que tels dans le champ du développement.

Cadre d'analyse

11 L'analyse privilégiera nécessairement une démarche orientée vers les acteurs dans leurs stratégies. Comme le souligne si bien N. Long, on a besoin pour comprendre le changement social, d'une approche plus dynamique qui mette l'accent sur l'interaction et la détermination mutuelle des facteurs et des relations "internes" et "externes" et qui reconnaissent le rôle central joué par l'action et la conscience humaine ${ }^{9}$. Se centrant d'abord sur l'étude de quelques trajectoires significatives, elle nous conduit de plainpied au cœur d'interfaces sociales particulières, où se jouent des stratégies diverses conduites par des acteurs déterminés.

Émergence des courtiers et contexte international

12 Si l'émergence des courtiers locaux du développement a été rendu aisée, c'est bien parce qu'elle a bénéficié d'un contexte international favorable. La crise persistante de l'Etat a conduit les bailleurs de fonds à revoir leur méthode dans la gestion de l'aide qu'ils octroient, notamment en diversifiant leurs interlocuteurs. Il est en effet facile de constater la tendance amorcée, ces dernières années, de verser directement l'aide au développement à des bénéficiaires identifiés sans toujours passer par l'Etat qui se trouve de fait de plus en plus contourné pour mauvaise gestion. Son inefficacité est décriée tant par les bailleurs de fonds que les acteurs sociaux qui interviennent plus directement dans l'action du développement. Cette démarche confère des ressources nouvelles à une société civile qui cherchait déjà à se structurer et de laquelle participent les diverses associations et ONG qui se créent. Cette fenêtre ouverte sur l'aide au développement, jusque là relativement bien contrôlée par l'Etat, libère une portion de l'aide, que les différentes associations cherchent à capter leur profit. Ainsi, au niveau de tous les grands bailleurs de fonds, s'ouvrent de nouveaux guichets où l'on négocie directement sans l'entremise de l'Etat, qui a dû partager, malgré lui, une de ses sources privilégiées de redistribution. L'apparition de ces acteurs, dans le champ du développement est donc un phénomène récent qu'il faudrait mettre en rapport avec les nouvelles orientations des bailleurs de fonds qui, de manière générale ouvrent des brèches vers une allocation plus directe des ressources qu'ils octroient au développement.

Une étude récente ${ }^{10}$ établit une chronologie relativement exhaustive du phénomène associatif au Niger. Cette chronologie fait apparaître l'existence de trois générations d'associations qui se sont développées, chacune à des périodes différentes, et qui montre bien l'émergence tardive de ces associations dites de développement, qu'on 
peut, sans problème, lier au contexte qui a été décrit plus haut. Cette chronologie fait apparaître les étapes suivantes :

- de 1960 à 1985, le mouvement associatif autonome est un phénomène marginal. les regroupements sociaux sont essentiellement canalisés dans de grandes organisations para-étatiques. Les associations qui se créent sont essentiellement des associations de ressortissants Ètrangers (amicales, clubs, mutuelles) auxquelles il faut ajouter quelques associations à caractère professionnel, culturel ou sportif ${ }^{11}$.

- A partir de 1985/86, les ONG de développement font leur apparition. Alors qu'elles étaient 13 au Niger lorsque l'armée a pris le pouvoir en 1974, on comptait en 1993 115. L'étude identifie deux vagues dans cette poussée d'ONG : une première marquée par l'implantation au Niger de ramification nationales d'ONG internationales (IRED et SIX S s'implantent par exemple durant cette période) et la seconde, située en 1991/1992, voit la création d'une nouvelle vague d'ONG, spécifiquement nigériennes ${ }^{12}$.

- A partir de 1991, année de la conférence nationale, on voit apparaître, pour se superposer aux précédentes, une troisième génération. Cette dernière est plus ouverte aux problèmes de société puisqu'on y trouve des associations de lutte contre la toxicomanie, des associations de défense de droits de l'homme, des associations religieuses et surtout des associations de développement. discours englobant et non exclusif, elle s'active surtout à mobiliser les "touareg de souche noire". Une telle catégorisation parait bizarre et même choquante a priori, en raison de ses connotations racistes ${ }^{15}$. Elle renvoie cependant à des distinctions fort répandues dans le langage commun et qui réfèrent à des considérations stigmatisantes bien précises: bouzou en haoussa ou bella en zarma cumule une dimension phénotypique et un statut social, en l'occurrence, celui d'esclave ou de la condition servile, vécu par certains groupes sociaux dans les communautés touareg.

C'est donc autour de cet ethnonyne atypique, "touareg de souche noire" que les initiateurs $\mathrm{du}$ projet vont forger un contenu. Ils opèrent tout d'abord par dénonciation avant de définir les contours de leurs revendications. 
l'association, ces inégalités touchent directement les "touareg noirs". Pour Timidria, ces derniers sont marginalisés tant dans les communautés touareg où ils vivent dans des conditions inhumaines, que dans la communauté nigérienne, où leur accès aux ressources de l'Etat semble fortement limité. Il est ainsi proclamé dans le "Documentcadre des touareg de souche noire" 16 :

"Nous touareg du Niger de souche noire, devant l'insouciance des pouvoirs publics qui se sont succédés depuis l'indépendance dans notre pays, à l'égard de notre sort qui se résume par une marginalisation criante de notre communauté, les exactions et humiliations de tout genre, le mépris de nos frères de souche blanche à notre endroit, notre exclusion de la gestion des affaires politiques et administrative de notre cher pays, le tout érigé en système oppressif..." 17

Le même document fait état des manifestations de cette marginalisation sur les plans politique et administratif :

"De tout temps nos frères de souche blanche ont été les bénéficiaires privilégiés des différents régimes, car ils sont les seuls à être nommés ministres, préfets et cadres de commandement. Le pouvoir ignore-t-il notre poids démographique et la pléthore de compétence en notre sein au point de nous subordonner à une frange minoritaire ?"

\section{Le document relève en outre que}

"La politique d'exclusion tendant à endormir notre conscience a été savamment orchestrée dans le domaine éducatif et scolaire en nous fermant les portes des écoles... car nos prétendus chefs estiment que nous ne sommes bons que pour rester dans l'ignorance et la servitude. Sinon comment comprendre que des écoles nomades crées depuis, n'ont commencé à accueillir les enfants de touareg de souche noire qu'en $1965^{\prime \prime}$.

21 Au delà du document-cadre, le discours dénonciateur de Timidria ressort surtout lors des manifestions qu'organise l'association. Ainsi lors d'un séminaire organisé par l'association, plusieurs participants ont rapporté des faits révélant les situations qu'ils vivent. Tantôt les militants de l'association fustigent les discriminations dont ils sont l'objet quand ils sont pressentis pour occuper tel ou tel poste rétributeur. Un militant rapporte dans ce sens qu'il a été élu pour diriger, au titre de son arrondissement, une section de la Croix Rouge. Mais cette élection ne fut pas entérinée parce qu'il était "noir". Pour le chef de canton de ladite localité, rapporte t-il, jamais un noir n'occupera une telle position, tant qu'il sera au pouvoir. Un autre rapporte que la fonction de chef de cabinet ministériel a été proposé à un de ses camarades. Mais ce projet de nomination n'a pas abouti parce que disait-on, une telle charge ne peut être confié à un "bouzou".

Pour beaucoup de militants de Timidria, la démocratie dont on clame tant l'existence au Niger, n'a pas encore franchi les frontières de leurs régions "qui continuent de croupir sous le poids de la servitude et de l'esclavage". Et d'appeler le Niger à appliquer toutes les conventions internationales qui abolissent l'esclavage.

De manière générale, l'association dénonce la persistance de l'esclavage dans les sociétés touareg et les conséquences de cette persistance sur les conditions de vie des "touareg de souche noire". L'association fustige aussi le silence du gouvernement face à ces injustices dont ils dénoncent l'anachronisme dans un contexte international qui les réprouve de plus en plus. 
On peut déceler trois niveaux de signification: la dénomination, les objectifs, les groupes mobilisés.

La dénomination de l'association

Timidria veut dire en Tamajech Fraternité. Ce mot est d'ailleurs constamment présent dans la dénomination de l'association. Comme l'affirme El Hadj Ismael, un marabout, membre actif du bureau de la section de Timidria à Maradi,

"ce que Timidria veut dire, c'est toi et moi, nous devenons frère. Que nous devenons Timidria, veut dire que nous devenons frère, que nous nous connaissions ou non. Et quand on devient frères, on se concerte. Chacun contribue à sa manière" 18 . Ici, la fraternité ne saurait se limiter à un simple slogan de principe. Elle constitue le symétrique inverse des dénonciations proférées par l'association à l'égard des inégalités marginalisantes observées dans leurs sociétés. Cette idée de fraternité puise son fonds légitimateur aussi bien dans l'islam que dans la constitution de la république et les conventions internationales sur les droits de l'homme, à travers notamment le principe de l'égalité et de l'universalisme Elle se traduit sur le plan de la production idéologique par cette pétition

"Il faut désormais, en parlant des touareg, ne plus faire état des distinctions sociales qui les spécifient, comme nobles, guerriers, affranchis, esclaves, etc., mais les nommer par Kel Tamajech, tout court, c'est «ceux qui parlent la langue Tamajech»."

Les objectifs de l'association.

L'association Timidria vise deux objectifs: la défense des droits de l'homme et le développement économique et social des zones nomades et semi-nomades du Niger ${ }^{19}$. Toutefois, il serait possible, si l'on s'en tient aux statuts de Timidria, d'identifier trois types d'ambition à travers les objectifs qu'elle affiche ${ }^{20}$ :

- il y a ce qu'on pourrait appeler les objectifs obligés, ceux que toute association est obligée d'afficher si elle veut se conformer au droit positif et à la morale politique dominante. Ce type d'objectifs conditionne implicitement sa reconnaissance. Chez Timidria, ils sont mis en forme à travers des préceptes classiques et devenus incontournables : "la sauvegarde de l'unité nationale par tous les moyens" ; "veiller à une cohabitation pacifique de toutes les communautés nigériennes", etc.

- il y a ensuite les objectifs qui spécifient l'association dans son identité particulière. Ici Timidria les met en forme à travers les principes ci-après : "Combattre l'arbitraire, l'exclusion, l'intolérance ou toutes formes d'atteinte aux libertés politiques et aux droits de l'homme" ; "œuvrer pour le progrès social et économique de ses adhérents" ; "sensibiliser ses militants sur leurs aspirations" ; "organiser des rencontres périodiques avec les autorités administratives et politiques en vue d'expliquer les aspirations réelles de ses membres aux plans politique, social et économique" ; lutter contre toutes forme de discrimination (racisme, régionalisme, ethnocentrisme). Cette catégorie d'objectifs permet d'identifier les enjeux autour desquels Timidria veut axer son militantisme associatif.

- il y a enfin une troisième catégorie d'objectifs qui sont ceux qu'on pourrait appeler les objectifs de développement. Dans les statuts de Timidria, il est dit clairement que l'association doit "rechercher les financements des projets et assurer leur suivi au profit des populations concernées". C'est sans doute ce type d'objectifs qui a permis de faire classer Timidria parmi les ONG de développement. Et on peut considérer par ailleurs cette dernière catégorie d'objectifs chez Timidria conditionne l'accomplissement de la seconde catégorie d'objectifs.

L'espace social à mobiliser 
Comme on l'a vu cet espace qui correspond dans le discours de Timidria aux touareg dit de "souche noire", renvoie à des groupes sociaux bien déterminés. Touareg de souche noire, c'est la catégorie descriptive construite par Timidria pour caractériser sa base ${ }^{21}$.

Pour Timidria, tous les touareg de souche noire sont, à tort ou à raison, assimilés à des gens de condition servile, de "condition inférieure" ou encore de "basse extraction". Autant de qualificatifs perçus par les idéologues de l'association comme de nature à les maintenir dans une situation de marginalisation au sein de leur propre communauté mais aussi au sein de la communauté nigérienne.

31 Ce schisme au sein de la communauté touareg, tel que Timidria le fait voir, rompt avec les perceptions homogénéisantes de la communauté touareg, souvent limitée aux "hommes bleus" du désert par une certaine hagiographie à but touristique. Or le discours de Timidria nous ramène à d'autres réalités mettant plutôt en avant une division de la communauté touareg, en touareg de souche blanche présentés comme dominants et ayant les faveurs du pouvoir ${ }^{22}$, et touareg de souche noire présentés comme dominés et vivant dans des conditions de servilité. sein de cette communauté une division sociale basée sur deux catégories : il distingue d'un coté les hommes libres désignés par le terme tamasheq ilellan et d'un autre coté la classe servile, iklan en tamashaq. Ces deux catégories recouvrent toute une hiérarchie d'échelons intermédiaires. Parmi les hommes libres, Bemus classe l'aristocratie guerrière (Imajeghân), les vassaux ou tributaires (Imghad), les religieux (Ineslemân), les artisans (inadan), mais aussi les métis constitués en tribus (Iboghollitân) et les affranchis (Iderfan, Ighawellân). Dans la dernière strate viennent les captifs (Iklan) ${ }^{24}$, qui se distinguent aussi par la couleur de leur peau. Mais comme le dit si bien Boubou Hama, "ce n'est pas un nom, c'est un état, une condition sociale exprimée par le mépris" ${ }^{25}$. A propos de mépris, J.P. Olivier de Sardan observe pour l'Ouest du Niger que "les bella sont perçus par les sédentaires comme la plus méprisable des ethnies ou des catégories sociales, et sont victimes de tous les ethnotypes du racisme (voleurs, sales, menteurs, sauvages...)" ${ }^{26}$. Et ce n'est pas sans mépris non plus, que ces touareg stigmatisés au sein de leur propre groupe sont perçus par la plupart des groupes ethniques du pays, comme des hommes inférieurs. En témoigne l'existence et la persistance des sobriquets tels que bella et bouzou rapportés plus haut.

C'est en fait à ce dernier groupe que Timidria cherche à inventer une identité et c'est en son sein qu'elle recrute ses militants les plus nombreux. Les Iklan (bella en songhay et en zarma et bouzou en haoussa), qui sont la raison d'être de Timidria, constituent au sein des sociétés touareg des groupes sociaux à part, caractérisés par leur ancienne condition de captivité. Selon Bemus, ils étaient razziés lors de guerres en zone sédentaire et étaient ensuite répartis dans les différentes familles Imajeghân, Imgdan ou Ineslemân en guise de récompense suite à leurs exploits guerriers. Toutefois, "aucune politique de fusion n'était pratiquée, mais seulement une politique d'assimilation culturelle qui s'accomplissait au bout de deux ou trois générations. Les petites filles étaient prises par les familles pour s'occuper des tâches domestiques, les petits garçons étaient initiés aux techniques pastorales : intégrés à la vie familiale de leurs maîtres, séparés de leurs parents, ils apprenaient rapidement le Tamasheq et acquéraient tous les usages de la société qui les avait incorporés. Bien que devenu partie intégrante de la société touareg, où ils jouaient un rôle économique prépondérant, ils ne se mélangeaient que très rarement par mariage à la classe des 
hommes libres. Ils se disent eux-mêmes Kel Tamasheq et ont conscience de faire partie de cette société dont ils constituent un élément moteur" ${ }^{27}$. Le propriétaire de l'akli (sing.) pouvait en disposer à sa guise. Il vit sous la dépendance d'un maître précis et travaille à son bénéfice à longueur d'année ${ }^{28}$.

Cette ethnographie n'a rien de surannée. Elle reflète à bien des égards la situation aujourd'hui encore de ceux que Timidria appelle les "touareg de souche noire", Il n'y a certes plus de razzia pour gonfler leur nombre depuis la fin du XIXème siècle qui marque le début de la colonisation française (monopolisation de la violence par l'État colonial et post colonial), mais, dans une large mesure, les sociétés touareg ont maintenu leurs structures qui ont continué de se reproduire en maintenant ces groupes dans des conditions de dépendance et parfois même d'esclavage sur des bases totalement passéistes qui s'accommodent mal aujourd'hui avec la forme républicaine de l'État nigérien auquel ils appartiennent. Cependant, le rapport établi ici entre Timidria et l'espace social qu'elle cherche à mobiliser, n'est jamais clairement apparent dans le discours de l'association. Il est déduit de la combinaison de deux idées-forces de Timidria, à savoir, touareg de souche noire et servitude. L'une renvoie à une distinction de type racial et l'autre à une distinction de type social. La notion de touareg de souche noire, tel qu'elle est utilisée par l'association, nous plonge en effet dans des divisions de type racial rappelant les Iklan d'origine négro-africaines qui vivent des conditions de servitude au sein des sociétés touareg ${ }^{29}$.

Il faut toutefois se garder de faire des généralisations hâtives qui auraient tendance à assimiler tous les touareg noirs ou encore tous les militants de Timidria à des Iklan.

Comme l'ont rapporté certains auteurs, dans les sociétés touareg, la couleur de la peau n'est pas le seul déterminant de l'identité sociale ${ }^{30}$. On peut dès lors suggérer que la notion de "touareg de souche noire" s'identifie certes aux populations touareg noires mais qu'elle se limite à celles qui socialement vivent dans des conditions de servitude ou qui sont marginalisées en raison de son origine servile. Ainsi distinction raciale et distinction sociale sont couplées pour construire la catégorie sociale que Timidria cherche à mobiliser ${ }^{31}$.

37 Cette catégorie ne constitue pas une population homogène. Elle ne correspond pas à un espace géographique continu. On ne peut pas dire non plus qu'elle se réclame des mêmes traditions historiques en raison de sa dispersion sur une grande partie du territoire nigérien. Par ailleurs, son analyse ne peut faire l'économie des transformations induites aussi bien par les politiques de scolarisation et la mise en place d'une fonction publique, que par les phénomènes de migrations qui ont suivi les multiples sécheresses qui ont frappé le Sahel pendant ces dernières décennies. On peut ainsi identifier dans l'espace social mobilisé par Timidria deux groupes touareg bien distincts aussi bien dans leurs ressources que leur localisation géographique.

Ainsi, le premier groupe est repérable dans la plupart des régions du Niger habitées dans les populations touareg ${ }^{32}$. On sait que compte tenu de leur stratification, les communautés touareg, surtout celles de l'Azawagh (cette région est fortement stigmatisée dans le discours des militants de Timidria) mais pas seulement, maintiennent toujours en leur sein certains groupes sociaux dans une condition de dépendance domestique ${ }^{33}$. Il ne s'agit point de survivance idéologique comme ça pourrait être le cas dans d'autres groupes ethniques du Niger, mais bien de condition 
servile vécue par certains groupes sociaux. A propos du second groupe, on peut le subdiviser en deux catégories :

- la première catégorie comprend un groupe d'importance grandissante établi dans les centres urbains en raison des sécheresses successives qui ont sévi ces dernières décennies au Niger ${ }^{34}$. On les repère dans la plupart des villes. On notera d'ailleurs dans ce groupe qui squatte les périphéries des villes, l'existence de communautés nomades non exclusivement touareg. On y trouve en effet aussi des communautés peul.

- La seconde catégorie comprend les touareg scolarisés qui se sont retrouvés à l'issue de leur cycle à des échelles diverses de l'administration publique. C'est en leur sein qu'on identifie les membres fondateurs de Timidria. Leur leitmotiv l'exclusion et la marginalisation dont ils sont victimes dans la répartition des postes de la haute fonction publique. Pour eux, il y aurait une discrimination à leur encontre qui les met à l'écart du développement de la société nigérienne de manière générale.

Ainsi, on peut considérer que l'espace social que Timidria cherche à construire, bien qu'uni par la langue, est disparate et en outre, qu'il n'est pas établi sur un espace géographique continu. Mais Timidria l'unifie autour des problèmes sociaux qu'il vit. En fait, il s'agit de deux problèmes cumulés mais différents. D'abord un problème d'esclavage résiduel et ensuite un problème de racisme. Ici le noyau fondateur de l'association saisit l'opportunité de la démocratisation pour tenter de faire élargir l'espace de la république et de ses lois. L'action qu'il conduit l'emmène d'abord à convaincre le groupe qu'il veut mobiliser, à le sensibiliser, à lui faire découvrir ses droits, et à initier en son nom des projets de développement. Ici la construction de sens prend plusieurs formes: définir une catégorie, lui donner un contenu, définir les activités à mener hors de la communauté façonnée. Il est intéressant ici de relever un mot couramment utilisé par l'association: la sensibilisation. Ce mot suggère que le groupe construit ne connaît pas toujours ses besoins. C'est l'association qui les définit et qui lui en montre la signification. Cohen et Comaroff caractérisent assez bien cette catégorisation et cette construction de sens quand ils disent que le courtier doit lui-même provoquer les besoins de ses clients potentiels. Il y a lieu d'abord de souligner que le courtier provoque lui-même les besoins qui se traduisent en informations à manipuler. Ensuite ses clients, entendons par là en l'occurrence les « touareg de souche noire ", sont potentiels, ce qui veut dire qu'ils n'existent pas, du moins au départ en tant que groupe social homogène. Le travail du courtier, quand il est dans sa phase de démarrage consiste donc à dépasser leur aspect virtuel.

La conduite du projet collectif : le travail associatif

C'est le travail associatif qui permet au courtier d'acquérir les ressources indispensable à son action. Ce travail est multiforme et constitue en cela autant de moyens qui lui permettent d'enrichir et de conforter son capital d'expérience.

Organisation de la structure et travail de coordination

41 L'association est d'abord le produit de l'action d'un groupe déterminé. Ce groupe, pour conforter la structure de l'organisation qu'il met en place a d'abord cherché à l'implanter un peu partout dans le pays. C'est un travail continu qui l'oblige à effectuer souvent des déplacements, à prendre des contacts et à convaincre.

Les dirigeants de l'association s'adonnent ensuite à un travail de coordination des activités dès qu'elles sont mises en place. Là encore, ils développent des capacités de rédaction, mais aussi de communication avec les sections et sous-sections qu'ils ont installées. C'est ainsi qu'ils initient à la correspondance administrative et aux missions 
"sur le terrain". Ils développent aussi des capacités de mobilisation de ressources financières pour permettre à l'organisation de fonctionner.

L'organisation de la structure et le travail de coordination permet à certains membres de l'association de développer des capacités organisationnelles, à mesure que leurs activités prend de l'ampleur. Ces compétences nouvelles confortent leurs positions dans la structure, car ils détiennent des informations, qu'ils contrôlent et diffusent selon le jugement qu'ils font des situations. Ils développent ainsi des capacités stratégiques fort utiles dans la gestion de leur pouvoir nouvellement acquis.

La prise de parole : acquisition de compétence rhétorique

Parmi les effets de la démocratisation dans les pays africains, on relèvera indiscutablement la multiplicité des espaces d'expression ouverts pour les partis politiques et les associations. En effets, sur des enjeux déterminés, ces organisations sont souvent sollicitées pour émettre leur avis. Ainsi, les dirigeants de Timidria, notamment son président, interviennent régulièrement à la télévision et à la radio. Ils rédigent et envoient des communiqués à la presse écrite. Ces espaces médiatiques sont des lieux privilégiés où ils apprennent à s'exprimer en public et à défendre des positions, renforçant ainsi leurs capacités à débattre. Ils acquièrent ainsi des compétences rhétoriques utiles à la conduite de leur militantisme.

Mais il n'y a pas que les media qui favorisent cette socialisation. Pour le cas de Timidria, il est clair que la participation de certains de ses membres à certaines rencontres internationales ${ }^{35}$, ou encore aux séminaires organisés par certains organismes installés à Niamey constitue un moyen efficace d'apprentissage de la prise de parole publique. Participent également de cette formation les tournées de sensibilisation qui permettent à certains dirigeants de l'association de prendre des contacts périodiques avec les militants de base qu'ils rencontrent lors des assemblées générales et des meetings.

Les actions de courtage : ressources mobilisées et interface à géométrie variable

Les actions de courtage que nous avons observées embrassent différents domaines.

Elles sont censées avoir été identifiées en fonction des besoins supposés des populations et construits par l'association qui se présente comme porte-parole et médiateur attitré. Ces actions sont ensuite soumises pour financement et mises en œuvre auprès des bailleurs de fonds ou pour satisfaction auprès de l'État. L'association traduit ainsi, selon la belle expression de P.J. Laurent, "les besoins des uns en projets pour les autres, moyennant une rente à négocier" ${ }^{36}$, car comme le montre bien Cohen et Comaroff, "the broker translates the unknown and inaccessible into the familar and the possible" ${ }^{37}$.

48 L'action menée par Timidria vers les bailleurs de fonds, qui servira de base à notre analyse, prend forme dans la recherche du financement des projets qu'elle définit. Dans ce cadre, des projets sont définis par Timidria. Elle les soumet par la suite aux bailleurs de fonds qui acceptent ou non de les financer. Plusieurs de ces actions ont déjà abouti. Relevons le financement par l'USAID d'un séminaire sur les droits de l'homme dans les zones nomades et semi-nomades et d'une caisse populaire d'épargne dans la commune de Maradi. Toujours dans la même localité, la coopération suisse a financé une coopérative de couture mise en place par un groupement féminin de Timidria. L'association prise dans sa globalité peut en effet être considérée comme une interface sociale au sens où l'entend $\mathrm{N}$. Long, c'est-à-dire, "a critical point of intersection or linkage between different social systems, a field or level of social order where structural discontinuities, based upon differences of normative values and social 
interests (meet]..." 38. Les systèmes sociaux qu'elle permet de relier montrent une extrême diversité. Timidria se veut porte-parole des touareg noirs, mais comme nous allons le voir ce groupe a lui-même historiquement généré d'autres sous-groupes, qui en font un ensemble composite impliquant de la part de l'association des mises en rapport avec des systèmes sociaux spécifiques. Autrement dit, il n'y a pas d'un côté le groupe des touareg noirs et de l'autre les bailleurs de fonds. C'est bien plus complexe que ne le laisse paraître cette opposition. L'interface a une configuration à géométrie variable, mobilisant pour chaque action de courtage des acteurs distincts.

Le séminaire sur "Les droits de l'homme en zone nomades et semi nomades"

Financé par l'USAID, il s'est finalement tenu à Tahoua du 26 au 28 octobre 1994. Initialement, il devait se tenir en septembre de la même année, mais les procédures financières ont été plus lentes que prévues. La tenue du séminaire est l'aboutissement de plusieurs mois de travail continu.

Il a fallu d'abord avoir l'idée de ce séminaire. Les bailleurs de fonds en organisent pour familiariser leurs partenaires nationaux avec l'esprit d'un projet ${ }^{39}$. Pour Timidria, l'organisation d'un tel séminaire est une gageure. L'association est jeune, elle est née en 1991. Ses animateurs le sont également. Leur âge tourne autour de la trentaine. En outre, ce ne sont pas des hauts fonctionnaires ayant choisi la voie associative comme moyen de reconversion après avoir occupé de hauts emplois publics ${ }^{40}$. Ils ne sont pas non plus des familiers de la machine étatique pour avoir été longtemps fonctionnaires. Les acteurs qui nous intéressent ici sont de nouveaux fonctionnaires et n'occupent pas des positions de pouvoir dans l'administration. Ils comptent, pour obtenir du financement, sur la pertinence de leur projet, sur la justesse de leur vue. En optant pour les droits de l'homme comme thème de leur séminaire, ils ont conscience que la tâche sera ardue. Car, en matière de droits de l'homme, il y a sur la scène des associations solidement implantées et bien insérées dans le circuit du financement international. En témoigne la fréquence de leurs activités et surtout leur médiatisation ${ }^{41}$. Ils ont aussi conscience qu'ils courent le risque de s'entendre dire par leur bailleur potentiel : "Arrangez-vous avec les associations de droits de l'homme de la place, elles ont plus d'expérience que vous dans ce domaine". Et c'est vrai que pendant la conférence nationale, ils étaient installés dans les tribunes du palais du sport à côté des autres associations de droits de l'homme. Mais ils se lancèrent quand même dans cette entreprise qui avait toutes les apparences des causes perdus d'avance.

51 Une fois le thème retenu, il fallait le définir. De quoi vont-ils parler à ce séminaire et qui va en parler? C'est ainsi que des thèmes de discussion furent retenus. Ces thèmes devaient refléter les préoccupations des populations qu'ils veulent représenter :

- les droits de l'homme en zones nomades et semi-nomades,

- les droits de la femme, de l'enfant en zones nomades et semi-nomades,

- les relations agriculteurs et éleveurs,

- l'éducation en zones nomades et semi-nomades,

- l'unité nationale.

Pour chacun de ses thèmes, ils avaient l'intention de choisir leur conférencier parmi ceux qu'ils estimaient être les plus compétents sur la place.

Forts de tous ces éléments, ils commencent la course au financement qui, de manière inattendue, aboutit rapidement. Auparavant, quelques membres du bureau se font recevoir par une personnalité importante de l'ambassade des États-Unis. Cette dernière 
s'est montrée sensible à leur thème et avait promis de "faire quelque chose". C'est l'expression consacrée.

Mais un accord de principe ne suffit pas pour obtenir un financement. Il faut finaliser le document de projet que les toutes les parties vont parapher. Les parties, ici, font référence à l'USAID et Timidria. Les représentants de l'association doivent ici s'initier au travail de rédaction et se familiariser avec le monde du financement $d u$ développement. En l'occurrence, ce monde prend forme à travers les structures de l'USAID.

Une fois le document signé, commence la phase de l'exécution du projet, qui correspond au début de l'organisation du séminaire. A ce niveau, les activités sont nombreuses :

- identifier les conférenciers et confirmer les dates fixées pour la tenue du séminaire ;

- le séminaire ne se déroulant à Niamey mais à Tahoua, il fallut prendre contact avec les autorités régionales, pour garantir leur participation à l'ouverture et à la clôture du séminaire, mais aussi négocier le lieu où se déroulera le séminaire et l'hébergement des participants. Ce dernier nécessitant un contact avec les opérateurs économiques de la place, notamment les hôteliers;

- faire acheminer à Tahoua les participants qui vont venir de tous les coins du Niger où l'association dispose d'une structure ;

- organiser le séminaire. C'est une tâche des plus difficile. Il faut veiller à ce que tout se déroulait normalement pour les cinq jours de présence à Tahoua. Que les participants soient bien installés, que l'amphithéâtre mis à la disposition du séminaire par la mairie soit bien nettoyé, les chaises installés en quantité suffisante, l'acoustique en état de marche.

Pour ce séminaire, l'association a fait venir à Tahoua plus d'une centaine de participants, tous normalement hébergés, et percevant des per diem gracieusement octroyés par l'USAID. Les organisateurs eux bénéficient de per diem plus élevés que les participants en leur qualité d'organisateurs.

Durant le séminaire, les travaux se déroulent sous le mode exposé-discussion. Il fallait aussi le faire couvrir par la presse régionale et nationale. Ces événements furent quotidiennement rapportés par la presse écrite, la radio et la télévision. Dans leur reportage, les journalistes ont régulièrement rapporté les propos du président de l'association dont le nom fut alors cité es qualité. Le séminaire se termine par une soirée culturelle à la maison des jeunes et de la culture de Tahoua.

Après le séminaire, il fallait rédiger le rapport du séminaire qui comprenait aussi un volet financier. Il fallait tout justifier pour bénéficier des derniers décaissements qui devaient de payer les indemnités. Ce travail sera effectué par les principaux organisateurs du séminaire.

Il fallait aussi organiser le départ des participants afin que tout se déroulât normalement.

60 Tout cela se déroule en présence d'un agent de l'USAID qui tenait une caisse dont l'ordonnateur était un membre de l'association, spécialement désigné par ses pairs, membres du bureau.

61 Ce projet a été négocié à Niamey par les membres de l'association et mis en œuvre à Tahoua. Le courtage en développement ici, a eu Niamey pour scène d'expression, bien que le séminaire se soit tenu à Tahoua. Ni l'association, ni son bureau, ont été en rapport avec l'ambassade américaine, mais plutôt quelques membres du bureau 
cooptés par leur pairs pour conduire le projet à son terme. En l'occurrence, ce furent le président de l'association et son secrétaire général qui furent mandatés pour conduite ces négociations. C'est ceux-là qui deviennent courtiers en développement par le travail de traduction qu'ils font des besoins supposés de leur militants en un projet à soumettre aux bailleurs de fonds. La plupart des bailleurs de fonds de la place ont été saisis pour le financement du projet. Mais un seul a donné une suite favorable à la requête de l'association. La configuration de l'interface se réduit au nombre des courtiers qui conduisent l'action. Quant aux systèmes sociaux qu'ils lient, ils comprennent d'un côté l'association dans son expression la plus globale, compte tenu du thème du séminaire et du public mobilisé (toutes les sections ont été représentées et les résultats du séminaire doivent être diffusés vers tous les membres de Timidria), et de l'autre coté un bailleur de fonds, à savoir l'USAID.

Le groupement féminin de Maradi

Il est intéressant ici de reprendre in verbatim les propos tenus par le secrétaire général de la section Timidria de Maradi :

"Nous avons commencé par les activités culturelles. Nous avons réfléchi, nous avons réalisé que c'était insuffisant. On s'est dit qu'il faut faire des choses concrètes, quelque chose d'utile pour nos gens qui souffrent beaucoup et dont le problème est de ne pas avoir de métier. Nous savons qu'il faut faire en sorte qu'ils aient quelque chose à faire. C'est ainsi que nous avons initié les projets de travaux manuels. Il y a la couture, le tissage, la maroquinerie. Nous avons fait un projet et nous l'avons transmis au Bureau Exécutif National (BEN); nous l'avons déposé partout, à la préfecture, à la direction de l'artisanat. Nous avons dit au BEN de le déposer partout dans les ambassades, le Canada, la Suisse. Notre ami que voici (il le désigne du doigt) est allé à un séminaire à Tahoua. Il y rencontra la coopération suisse. Il leur parla de la similitude de leurs objectifs et du document préparé par Timidria Maradi. La coopération suisse demanda qu'une copie du document lui soit envoyée. Ce fut fait dès qu'il revint du séminaire. Dès qu'ils ont reçu le document, ils ont admis que le document va dans le sens de leurs objectifs. Ils ont alors demandé à nous contacter pour étudier les possibilités de coopération. Ils sont venus ici. Nous les avons convaincus. En fait notre requête couvrait quatre à cinq volets. C'est la coopération suisse qui proposa d'abord une phase expérimentale qu'ils financèrent. Dans cette phase la coopérative comprend 30 membres. Sila phase expérimentale réussit, les autres volets ont toutes les chances d'être financés, nous a garanti la coopération suisse. Beaucoup de gens viendront ainsi apprendre à travailler afin qu'ils puissent plus tard subvenir à leurs besoins sans problèmes et sans mendier". ${ }^{42}$

Le groupement féminin de Maradi a été mis en place peu après l'installation de la section locale de Timidria. Au départ, ce groupement devait réunir plusieurs femmes touareg noires en vue d'une formation à diverses activités ou de la valorisation de leurs capacités à des fins productives. En terme de réalisation, Timidria avait, avant la mise en place du projet suisse, procédé à l'inventaire des ressources disponibles au sein du groupement en vue de la recherche du financement nécessaire à sa mise en valeur à des fins productives. Plusieurs bailleurs de fonds avaient été saisis à cet effet. Que de promesses sans suite me disait le président de Timidria qui n'en restait pas moins optimiste sur la possibilité de "décrocher quelque chose". L'obtention du financement ne suivra pas la voie attendue, celle qui devait venir de Niamey. Elle suivra une voie détournée et aboutira à la mise en place d'un projet qui fait aujourd'hui la fierté de Timidria. 
64 La coopération suisse avait organisé un séminaire sur le thème "Genre et Développement" à Tahoua. Comme à l'accoutumée, ce type de séminaire rassemble les différents secteurs de l'activité étatique. Un membre de Timidria participera à ce séminaire non pas pour représenter son association mais en qualité d'agent de la direction départementale du service dont il relève. Le cours des discussions lors de ce séminaire l'a amené à parler du groupement mis en place par une association dont il est membre. Ses propos suscitèrent l'intérêt de la coopération suisse. Cette coopération délégua une mission à Maradi aussitôt après le séminaire. La mission conclut à un appui au groupement féminin Timidria. Cet appui devait se traduire par le financement d'un atelier de couture. La coopération suisse devait non seulement acheter des machines à coudre au groupement féminin mais assurer une formation en alphabétisation et en gestion coopérative aux membres du groupement.

En contrepartie, Timidria devait fournir les locaux qui allaient accueillir la coopérative. Une souscription fut lancée par le bureau au sein de la section pour mobiliser les fonds nécessaires à la construction de l'atelier. L'atelier fut bâti en séco par tous les membres de Timidria Maradi, pour la plupart des touareg noirs. L'argent réuni permit d'acheter le matériel nécessaire à la construction.

66 Ce n'est là que le début de l'action. Car il fallait concevoir le document de projet et le faire signer par toutes les parties concernées. Vint par la suite la phase d'exécution du projet. L'atelier de couture nécessite l'achat de machines à coudre, mais aussi de tables et de chaises. Le secrétaire général de la section Timidria de Maradi dut pour ce faire se rendre à Niamey pour visiter les boutiques qui en vendaient afin de se faire établir une facture proforma . Les machines et le mobilier furent achetées sur cette base. Un camion fut loué pour les acheminer à Maradi, au siège de la section locale de Timidria. La remise officielle du matériel eut lieu en présence des autorités locales, dont le préfet en personne. La télévision nationale rapporta l'évènement qui figura dans ses titres. Au niveau national, les journalistes louèrent cette nouvelle forme de coopération et surtout le sérieux de cette association qui réussissait à décrocher des financements internationaux.

Puis vient la phase de la formation. Elle comprend deux volets comme cela a été noté plus haut. Pour le volet alphabétisation, Timidria prit contact avec les services départementaux de l'alphabétisation. Un programme de formation fut défini et chiffré à l'attention de la coopération suisse qui le finança. Ainsi, tous les membres du bureau de la section locale de Timidria reçurent une formation. L'alphabétisation leur était nécessaire car la plupart d'entre eux ne savait ni lire ni écrire. C'est là une première phase dans l'exécution du projet.

Le second volet de la formation entre dans le cadre de la gestion coopérative. Celleci sera exécutée par les agents des services départementaux du plan qui étaient passés à l'occasion par Timidria. Cette formation avait été programmée pour les encadreurs de la coopérative. Ils ont ainsi appris la comptabilité, la tenue des cahiers de compte, le rapport avec les fournisseurs.

Quant au projet proprement-dit, il consiste à former à la couture les membres féminins de Timidria pour leur apprendre un métier. Les machines seraient ensuite à leur disposition pour leur commerce moyennant redevance à la coopérative.

Des contacts permanents sont maintenus entre la section Timidria et le responsable du projet à la coopération suisse. Désormais d'ailleurs, les femmes de Timidria participent 
aux séminaires nationaux qui concernent "Genre et Développement", où elles font part de leur expérience. Lors du rassemblement féminin qui eut lieu à Niamey à l'occasion de la Journée internationale de la femme, quatre femmes de la coopérative furent invitées par la coopération suisse à cette rencontre... Elles furent sélectionnées par l'association qui s'était en outre chargée d'organiser leur départ vers la capitale.

71 Ici aussi, comme ce fut le cas pour l'organisation du séminaire de Tahoua, il apparaît une autre configuration de l'interface liée aux systèmes sociaux qu'elle permet de mettre en rapport. Le public de Timidria comprend essentiellement une population touareg migrante occupant les nouveaux quartiers de Maradi comme Magama et Zarya. Cette population s'y est installée suite_aux sécheresses de 1974 et de $1984{ }^{43}$. La grande majorité de cette population n'a pas d'activité fixe. D'ailleurs les dirigeants de l'association font ressortir, pour décrire leur situation économique, "qu'ils ne travaillent pas, qu'ils sont marginaux, qu'ils mendient". Dans le département de Maradi, ce groupe de migrants ne constitue pas la seule population touareg. On en trouve au nord de Dakoro (arrondissement correspondant à la partie septentrionale du département), où l'on retrouve, selon l'association, des groupes "vivant dans des conditions de servilité révoltantes". Ceux-là ne sont pas concernés par le projet bien qu'ils constituent un des groupes-types qui légitime l'existence de Timidria. Du côté de la coopération suisse, ce sont les responsables chargés du programme "Genre et Développement", qui apparaissent sans cesse dans le discours de l'association. Ils ont provoqué le projet qu'ils ont intégré dans leur programme et concourent à sa mise en œuvre. Ainsi, la coopération suisse traduit-elle sa présence par une structure administrative bien précise. L'interface prend forme à travers les responsables de l'association chargés de suivre le projet au niveau local et qui sont aussi les interlocuteurs de la coopération suisse dans la gestion du projet.

La caisse d'épargne populaire Timidria de Maradi

72 Timidria, c'est le nom donné à une caisse populaire d'épargne qui a été mise en place par l'association du même nom. Là, l'idée de la mettre en place vint du secrétaire général de l'association. Il savait par le biais d'un de ses camarades qu'il existait un mouvement régional des caisses populaires. Ce mouvement initié par l'USAID rassemble plusieurs caisses populaires de par le monde. Au Niger, ce mouvement n'en est qu'à ses débuts. Son développement avait été favorisé par la faillite de la caisse nationale d'épargne, organisme d'État. Cette faillite avait occasionné beaucoup de panique chez les petits épargnants qui ne voulaient plus pratiquer l'épargne institutionnelle. Mais le projet de caisse populaire plaisait beaucoup au secrétaire général. Leur association Timidria a bien une caisse, qui à l'occasion, prêtait de l'argent aux membres. Ces derniers pouvaient ainsi s'adonner au petit commerce de détail, histoire de ne plus mendier ou ne plus vivre aux crochets d'autrui, comme c'était souvent le cas. L'idée de créer une caisse allait alléger considérablement les finances propres de Timidria. Et c'est comme cela qu'il se lança dans l'aventure.

73 Pour créer une caisse populaire agréée par l'USAID, il fallait rassembler au préalable au moins une cinquantaine de personnes. Ces dernières devaient donner une participation de départ d'au moins $2500 \mathrm{f} \mathrm{cfa}$. Le secrétaire général de l'association fit la proposition aux autres membres du bureau qui l'approuva. Il ne restait plus qu'à mobiliser les membres de Timidria à Maradi. Sur les registres de l'association, ils étaient plus de 200. Il y avait bien d'autres qui ne pouvaient pas cotiser en raison de leur situation économique, mais qui participaient aux activités de l'association. Ils arrivèrent non 
sans difficulté à mobiliser ainsi les 50 membres ainsi que leur cotisation. Par la suite, ils firent appel au mouvement national des caisses populaires qui vint ainsi installer officiellement la toute nouvelle caisse.

La création d'une caisse dans le cadre de ce mouvement entraînait automatiquement la location d'un local approprié et son équipement en mobilier à la charge de l'USAID.

Par ailleurs, le mouvement fournissait tous les registres, les carnets d'enregistrement, en somme tous les moyens de fonctionnement, dont le salaire d'un gestionnaire. La caisse est dotée d'un compte à la Banque Internationale pour l'Afrique de l'Ouest (BIAO). La caisse fait l'objet d'un contrôle périodique mensuel. Ce contrôle est exercé par le représentant du mouvement des caisses populaires. Timidria eut tous ces avantages. Aujourd'hui, il est facile de retrouver la caisse Timidria. Sis sur une des rares avenues bitumées de la ville de Maradi, n'importe quel chauffeur de taxi de la ville identifierait le siège pour y être passé au moins une fois par journée de travail. À Maradi, beaucoup de gens confondent l'association et la caisse.

76 La caisse Timidria a aujourd'hui à peu près 195 membres. Ils se réunissent régulièrement en assemblée générale. La caisse comprend en outre deux comités et un gestionnaire qui assure son administration.

77 Le secrétaire de la section, initiateur principal de la caisse, l'a déjà représentée à plusieurs réunions du mouvement hors de Maradi. Il a acquis de l'expérience. Il est souvent mandaté par le conseil d'administration de la caisse pour porter telle ou telle décision du conseil d'administration auprès du représentant de l'USAID.

78 Ici encore l'interface prend une forme particulière. L'association a favorisé la mise en place d'une caisse prévue au départ pour ses militants. Mais son rôle d'interface paraît limitée à la période de négociation du projet et n'implique pas une fois que la caisse a été instituée une mise en relation du bailleur qu'est l'USAID avec les membres de Timidria et cela, compte tenu du caractère autonome de la caisse. Il faut cependant reconnaître que cette paternité renforce sa position vis-à-vis de ses militants, et des bailleurs de fonds. On voit bien comment un rôle ponctuel d'interface se transforme en ressource pour la structure qui lui a servi de support, et, au sein de la structure pour les acteurs qui l'ont initiée.

Émergence et carrière du courtier : quelques biographies personnelles significatives

Les actions de courtage, bien qu'officiellement portées par l'association Timidria, sont en réalité le fait de quelques militants dynamiques à qui il revient à chaque fois d'assurer les fonctions d'intermédiation qui, à la longue, les transforment de fait en courtiers. Comment ces courtiers émergent-ils du lot des militants de l'association? Qu'est-ce qui les distingue des autres types de courtier en développement? Des éléments de réponse seront proposés après la présentation de quelques biographies personnelles significatives.

80 A est né en 1964. Administrateur à l'inspection du second degré sise sur la rive droite du fleuve, il était initialement professeur d'histoire et géographie. Avant d'être engagé dans ce premier emploi, il avait, après des études secondaires ayant débouché sur le diplôme de fin d'études normales, suivi les enseignement de la faculté de pédagogie d'où il obtint le diplôme de professeur de CEG. Professeur de C.E.G., il le sera quelques années avant de se faire affecter dans l'administration de l'éducation au niveau d'une inspection du second degré à Niamey. Depuis 1991, date de création de l'association Timidria qu'il préside, sa vie s'est profondément transformée. Cette dernière activité 
lui prend beaucoup de temps. Sans quitter sa profession dans l'administration de l'éducation nationale, il consacre beaucoup de temps à l'association dont les activités ne cessent de prendre de l'ampleur. Lui-même en a conscience comme le montrent bien les propos qu'il a tenus lors d'un entretien que nous avons eu avec lui ${ }^{44}$ :

"Au moment où il faut créer Timidria. dans la matinée, c'est toujours le déplacement, dans la soirée. c'est toujours le déplacement. dans la nuit. ce sont les réunions ou les réflexions jusqu'à 3 heures- 4 heures du matin, tout le temps en train d'écrire, fini ce calvaire de papier et la sensibilisation, et après, maintenant, c'est la sensibilisation auprès des ministères. des différents ministères. et maintenant, c'est la sensibilisation pour l'intérieur du pays. Il faut chercher un papier par là, rencontrer des gens ; pour aller à la maison, il faut 1 heure du matin". télévision à côté du préfet de Tahoua et d'un représentant de l'ambassade des États-Unis. On a parlé de lui dans les journaux. Et surtout ce séminaire lui a permis de se familiariser avec d'autres types d'activités: la rédaction, la rencontre avec les bailleurs de fonds pour expliquer son projet, l'élaboration de document de projets, les rapports avec les fournisseurs pour l'achat des équipements de bureau financé par l'USAID dans le cadre du financement du séminaire, des voyages d'études dans la sous-région etc. Autant d'activités auxquelles il n'était pas du tout préparé en sa qualité d'enseignant de profession. Mais pour lui, il fallait "se mouiller" s'il voulait donner à son association une envergure à laquelle les fonds propres de l'association ne pouvaient pas lui permettre d'accéder. Sa position sur la scène politique nationale s'est davantage confortée avec les forums de réconciliation qu'il a organisés à Tahoua et à Agadez.

82 I est journaliste. Il travaille à l'agence nigérienne de presse. C'est un homme discret mais qui donne l'impression "d'en vouloir". Après avoir fait son école primaire à Tchintabaraden, il poursuivit ses études secondaires à Tahoua, chef-lieu de département dont relève Tchintabaraden. Au CEG de Tahoua, il obtint un BEPC avant d'intégrer l'école de journalisme. Membre fondateur et militant actif de Timidria, il est secrétaire chargé de l'organisation. La première fois que nous nous sommes rencontrés après plusieurs rendez-vous non honorés, il était venu me confirmer mon invitation au séminaire de Tahoua. Il était chargé d'organiser les voyages à partir de Niamey. Il s'est rendu à Tahoua bien avant la date prévue pour la réunion, ceci afin de s'assurer que la salle serait prête dans les délais, que tous les participants du séminaire sont bien arrivés. Il était responsable de l'hébergement. Il assurait aussi la jonction avec le responsable financier de l'USAID, spécialement envoyé à Tahoua pour assurer l'exécution financière du projet. C'est aussi lui qui jouait le rôle d'ordonnateur des dépenses et de caissier. Au séminaire, on ne le voyait jamais dans la salle pendant les travaux du séminaire. Il n'apparaissait que pour donner des informations pratiques. Il était à coup sûr le coordinateur du séminaire, sa cheville ouvrière. On est bien loin du travail journalistique de rédaction de dépêche. Il fut présenté à la députation dans l'arrondissement de Tchintabaraden dont il est originaire par l'un des grands partis de la place lors des dernières élections législatives. Il ne fut pas élu.

B est né en 1964. Il est technicien supérieur de l'hydraulique. Il eut une scolarité primaire et secondaire normale à Illéla et à Tahoua qui a débouché sur un baccalauréat C. Après des études infructueuses en mathématique à l'Université de Niamey, BA fut retenu à l'ESTHER de Ouagadougou, dans un cycle de technicien supérieur en hydraulique. Pendant sa scolarité, il bénéficia d'une bourse du fonds français d'aide et de coopération. Après ces études il accomplit son service civique en qualité de 
professeur de math dans un collège ${ }^{45}$. Affecté plus tard à la direction départementale de l'hydraulique à Maradi, il se fera vite remarquer par son dynamisme et son esprit d'initiative. C'est ainsi qu'il participera à plusieurs séminaires nationaux à titre individuel mais parfois pour remplacer son directeur. Il représentera même ses collègues de la direction départementale au congrès de leur syndicat, à Niamey. B a eu l'opportunité de participer à un séminaire à Ouaga dans le cadre d'un perfectionnement professionnel. B n'est pas membre du bureau exécutif national de Timidria, ni même membre du bureau de la section de Maradi. Originaire de la région de Illéla dans la région de Tahoua, il ne maîtrise que très faiblement le tamasheq. Sa langue, c'est plutôt le haoussa. Lors d'un séminaire organisé par l'association, il était frappant de le voir commencer toujours ses interventions par ce prolégomène :

"Il faut que l'assistance m'excuse car je tiendrai mon propos en haoussa, car mon tamasheq n'est pas très solide pour me permettre de m'exprimer".

Il est venu à Timidria par l'insistance de certains de ses amis membres de l'association. Il y a adhéré aussi parce qu'il est convaincu que l'association se bat pour des causes justes. Son rôle dans l'association est devenu important en raison de son dynamisme et du rôle qu'il joue aussi bien dans la coopérative dont il est un des quatre encadreurs que dans la caisse populaire d'épargne dont il est membre fondateur et premier gestionnaire, poste qu'il lui a conféré la lourde tâche de la mettre en place. Aujourd'hui à Maradi, son nom est inséparable de Timidria.

Ag. est instituteur à l'école Magama dans la commune de Maradi. C'est dans cette commune cosmopolite qu'il est devenu le secrétaire général de la section Timidria de Maradi qu'il a contribué activement à installer. Originaire de Idouk, à 13 kilomètres de Tchintabaraden, il est né vers 1964. C'est à Tchintabaraden qu'il a passé sa scolarité primaire et secondaire. Après avoir eu son BEPC, il poursuivit ses études au cours normal de Tahoua ${ }^{46}$. Il y obtint un diplôme d'instituteur-adjoint. Ag servira dans plusieurs localité du Niger : Tillabéry, Téra, Gothèye, avant de venir à Maradi. Entre temps Ag eut le temps de se perfectionner en passant plusieurs concours : le BS 1 et BS 2 qui lui valurent de devenir instituteur. Ag se présente lui-même comme un battant. A Maradi, après des vacances scolaires au village, il se fit un devoir d'installer et d'animer la section locale de Timidria. Actif au sein de la section, il est encadreur au niveau de la coopérative. Comme B qu'il a emmené à Timidria, il est membre-fondateur de la caisse populaire d'épargne créée par l'association. C'est lui qui eut l'idée de la caisse et semble avoir été chargé de suivre plus spécialement les activités.

Comment est-on arrivé à détacher ces identités remarquables que sont devenues A, I, B, et $\mathrm{Ag}$ du groupe des autres militants de l'association? De manière générale, on peut dire que le personnage du courtier s'impose de lui-même dès lors qu'on évolue dans le milieu associatif. On peut distinguer au sein de l'association plusieurs types de militants. Il y a ceux qu'on pourrait appeler les points focaux. Ils constituent une espèce de point de ralliement de tous les militants actifs de l'association. Cette position, ils la détiennent en raison de leur âge ou d'autres types de ressources personnelles, comme la possession d'un domicile propre ou encore l'ouverture d'esprit ou des prédispositions à rendre leur maison accueillante, à faire en sorte que tous les militants qui viennent s'y sentent à l'aise, "comme chez eux". C'est par exemple, le cas de Ig qui occupe dans l'association le poste de secrétaire à l'information. Beaucoup de réunions du bureau se tiennent à son domicile. Mais Ig n'est alphabétisé qu'en arabe et connaît bien le Tifinah. Et sa connaissance de la langue française qu'il a appris "sur le tas" n'est 
pas solide. Mais cette faiblesse en langue française ne l'empêche guère de s'exprimer. Autant de facteurs qui ralentissent sa transformation en courtier (parce qu'il n'est pas exclu qu'il devienne lui aussi courtier) ou l'empêchent de le devenir. On peut encore identifier un autre type de personnage, qu'on peut qualifier d'idéologue: deux cas peuvent être rapportés pour illustrer cette situation. Même s'ils n'opèrent pas dans le même registre, leur rôle d'idéologues apparaît nettement à divers niveaux. Dans les réunions, ils interviennent souvent pour expliquer le sens de leur lutte. Leurs discours sont souvent accrédités d'une certaine force. C'est le cas de El hadj. Marabout de son état et grand orateur, c'est lui qui souvent quand on pose le problème de l'esclavage en appelle à l'islam, sourates à l'appui, pour montrer à ses camarades l'utilité de la foi en Dieu, qui ne fait aucune distinction entre les croyants. C'est lui m'a expliqué de la manière la plus convaincante ce que Timidria voulait dire. Il y a aussi Alk. Gardien dans une importante société d'économie mixte, il s'impose dans l'association comme un intellectuel. C'est un poète. Il a déjà rempli plusieurs cahiers d'écolier de ses poèmes si prisés par ses camarades qui aiment souvent les exhiber. Il n'écrit qu'en haoussa (avec caractères latins) et tamasheq (en tifinah). il fait la fierté de ses camarades. Il conclut toujours ses interventions par des poèmes toujours très bruyamment applaudis. Malgré leurs ressources personnelles indéniables, aucun de ses personnages n'a paru imposer sa présence en qualité de courtier dans les différentes activités décrites plus haut.

Ceux qui ont été retenus et dont les biographies personnelles ont été rapportées, comportent plusieurs spécificités qui méritent d'être relevées : Ils naviguent sans cesse entre un groupe dont ils définissent les contours précis au moment où ils conçoivent leur projet et le bailleur de fonds qui accepte de financer le projet. Au moment de la préparation du séminaire de Tahoua, ceux qui faisaient sans cesse la navette entre l'USAID et leurs camarades éparpillés dans tous le pays, ceux qui ont rédigé le document du projet et qui prenaient tous les contacts avec les fournisseurs, les autorités administratives, c'était $\mathrm{A}$ et $\mathrm{I}$. Au moment du déroulement du séminaire, ce furent encore eux qui assuraient les opérations comme le paiement des per diem après avoir fait les décaissements nécessaires auprès du bailleurs de fonds. C'étaient eux qui procédaient aux répartitions des tâches tout au long du séminaire. Après le séminaire, ils étaient chargés de la coordination de la rédaction du rapport final nécessaire pour obtenir les derniers décaissements. Leur position en qualité de courtier va en se confortant, puisque le séminaire, par la publicité qu'il a eu, leur a permis de se faire connaître. Beaucoup de leurs projets sont en voie d'aboutir. L'USAID voudrait leur financer un autre séminaire du même genre que celui de Tahoua. Et le Canada a, semble-il, accepté de financer des actions de sensibilisation autour des conflits agriculteurs/éleveurs dans la région de Maradi. A Niamey, on les connaît mieux depuis qu'ils ont organisé le séminaire.

Pour les projets de Maradi, avant de partir sur le terrain, une visite de reconnaissance rendue à la coopération suisse a permis de rencontrer le responsable du financement suisse de Timidria. Ce dernier m'a de tout suite suggéré d'aller rencontrer à Maradi B et Ag. C'est eux qu'il a désignés et pas d'autres. Il reconnaissait qu'ils étaient les principaux initiateurs du projet. Arrivé à Maradi, tout mon séjour fut encadré par eux, ensemble ou individuellement. Dans les réunions ils organisaient les débats. Ils étaient incontestablement les mieux informés de la vie de leur association et du fonctionnement de leur projet. Les militants de l'association à Maradi les reconnaissaient comme tels. Ils s'en remettaient volontiers à eux pour toute question qui pouvait leur être posée. Ces courtiers rencontraient les autorités administratives, 
ainsi que les bailleurs de fonds qui venaient à Maradi. Un jour, alors que j'étais dans le bureau de $\mathrm{B}$ il reçut un coup de téléphone de la coopération suisse à Niamey. Son interlocuteur lui demandait de sélectionner quatre femmes, membres du groupement féminin pour un séminaire qui devait se tenir dans la région de Niamey. Il rejoint tout de suite Ag afin qu'ils opèrent les choix nécessaires et organisent le voyage. Il leur fallait aussi financer les frais de voyage, prendre aussi contact avec les conjoints des femmes retenues pour qu'ils permettent la participation de leurs épouses au séminaire. Il leur fallait ensuite envoyer les noms à la coopération suisse, confirmer la participation des femmes retenues et organiser les modalités pratiques du voyage. Leur rôle de courtier s'exprime à chacun des niveaux de cette opération qui ne va sans leur conférer une certaine influence personnelle auprès de leurs camarades ainsi qu'auprès de la coopération suisse. Leur popularité, avec ce qu'elle impliquait en terme d'avantages divers (du fait de leur participation à des séminaires, ils percevaient des per diem, développaient leur réseau de relations) qui suscitent des convoitises chez certains militants, ce qui ne va pas sans susciter des conflits de tous genres. Le conflit autour d'un rôle conforte l'idée de l'existence de ce rôle.

Ces portraits nous révèlent plusieurs éléments qui semblent identifier les acteurs qui sont en train d'intégrer progressivement à leur rôle de militants associatifs des ressources constitutives du courtage en développement. Leur action n'est pas orientée vers leur espace de vie comme les courtiers décrits par G. Blundo ${ }^{47}$ qui se caractérisent par leur localisation et la position particulière qu'ils occupent dans le milieu vers lequel ils cherchent à attirer les projets de développement. Ils ne sont donc ni leaders d'associations villageoises, ni leaders d'organisations fédératives ${ }^{48}$. Par ailleurs, s'ils vivent en ville comme on l'a vu, ils n'ont pas le même profil que ces notables locaux décrit par P.T. Robinson qui occupent simultanément des positions fortes en ville et au village, l'une renforçant l'autre et vice-versa ${ }^{49}$. En outre, si dans leurs actions, ils ont beaucoup de points de ressemblance avec les courtiers décrits par Cohen et Comaroff ${ }^{50}$, il n'en reste pas moins que ces derniers cherchent à drainer des ressources étatiques alors que même les courtiers qui opèrent dans le cadre de Timidria s'intéressent surtout à des fonds extérieurs. Il est vrai les situations ne sont pas les mêmes. Dans le premier cas, l'État dispose de ressources que le courtier tente de drainer vers un espace social donné, alors dans le second cas, il les attend presque totalement de l'aide extérieure.

Ceux qui sont devenus courtiers par le biais de leurs activités au sein de l'association Timidria sont avant tout fonctionnaires de l'État, donc détenteurs d'un capital scolaire sont au sein de l'association Timidria des membres actifs à l'exception de B qui, bien que ne faisant pas parti du bureau de sa section, en reste un membre actif à travers les activités diverses qu'il anime et qui en font en réalité un élément essentiel dans la structure de l'association non seulement dans sa section de rattachement à Maradi mais aussi au niveau du siège de l'association à Niamey. Ils s'identifient beaucoup au groupe qu'ils ont construit, celui des touareg de souche noire. Mais nous avons vu que ce groupe constituait une réalité hétéroclite qu'il n'est pas possible de localiser dans un espace géographique bien circonscrit. Eux vivent en ville et émergent au fur et mesure que l'action de l'association prend de l'ampleur.

91 Cette activité les occupe beaucoup, bien qu'elle ne soit que secondaire pour le moment, car tous maintiennent encore leur activité principale. Ils lui consacrent beaucoup de temps. Timidria n'attend donc pas des subsides de l'État pour vivre. Les cotisations des 
membres quoiqu'importantes en raison de leur nombre, ne sauraient non plus suffire à entreprendre des actions de grande envergure. La recherche de fonds extérieures devient pour eux une sorte de passage obligé s'ils veulent conforter leur position dans l'association et accroître leur stock de relations personnelles. Aussi sont-ils obligés de développer des compétences nouvelles leur permettant d'émarger à ces nouveaux guichets du développement. Ils acquièrent ainsi une meilleure maîtrise des rouages de l'État et des hommes chargés de l'animer. Ils développent aussi des compétences organisationnelles indispensables à la mise en œuvre des actions de courtage tout comme ils développent des capacités stratégiques ainsi que des capacités de négociation, à mesure que leurs activités prennent de l'ampleur.

Ils deviennent ainsi courtiers par la force des choses, malgré eux. Au départ, devenir courtier n'a pas été programmé. Quand l'association se mettait en place, ses membres actifs ne s'engageaient que pour chercher des fonds ou pour servir d'intermédiaires entre des développeurs et leurs populations cibles. Ils ont très vite fait de comprendre qu'une association ne survit pas quand on ne parle pas d'elle, Elle doit nécessairement produire des activités concrètes au profit de ses membres. C'est ainsi qu'il leur a fallu fréquenter régulièrement les ministères pour faire des connaissances ou activer celles qui existent déjà. Il fallait aussi se faire connaître au niveau des ambassades et des organismes de développement en envoyant des prospectus et aussi en s'y présentant physiquement. Les copains, amis et sympathisants des ministères peuvent être à l'occasion de bons informateurs qui renseignent sur les opportunités de financement. Ils connaissent les filières. Ils ont aussi du papier et des photocopieuses qui permettent de multiplier des dossiers.

Avec les bailleurs de fonds, ils se familiarisent avec les documents de projets, ces fameux formulaires qu'il faut savoir remplir correctement si l'on veut accéder à la rente. Il faut aussi connaître les deskmen, ces "super commis du développement" qui ont la tâche d'identifier les projets et les ressources humaines nécessaires pour les mettre en œuvre, maintenant que cette activité ne relève plus de l'État. Pour ces gens-là, Timidria est une association comme une autre qui peut servir la politique de leur employeur. Ainsi, se familiariser avec le monde des associations, c'est surtout renforcer ses ressources professionnelles propres, puisque s'ouvrir aux associations correspond à la nouvelle politique désormais prônée. Pour eux, le contact avec les associations, permet d'approfondir la connaissance de leur terrain. Plus ils connaissent leur terrain, plus ils sont performants aux yeux de leur supérieur hiérarchique, car connaître son terrain, c'est savoir identifier les bonnes associations, celles-là même qui sont capables de réaliser les projets nés de la nouvelle orientation et qu'on peut exhiber lors des réunions internationales pour illustrer les trouvailles en matière de coopération au développement. Connaître les bonnes associations renforce la position qu'ils ont acquise dans leur organisation.

Pour les associations, la réalisation d'un projet, c'est à dire sa bonne exécution, entraîne automatiquement une nouvelle requête, souvent provoquée par le bailleur de fonds, qui fait miroiter de nouvelles possibilités de financement. Les bonnes exécutions répétées de projets permettent d'acquérir une nouvelle compétence dans l'exécution des projets et de "se faire un nom" dans le monde du développement. Car, dans ce monde là, plus on vous connait, plus on est prêt à vous financer les projets que vous proposez. Plus vous mobilisez des financements, plus sur votre espace social d'action, vous acquérez une nouvelle notoriété et une nouvelle légitimité qui iront grandissantes 
à mesure que vous réalisez de nouveaux projets de développement. Les courtiers en arrivent à occuper ainsi des positions confortables et convertibles sur le plan politique comme on l'a vu avec I et comme le montre les cours assidues que font les partis politiques à ces militants d'association devenus maîtres dans l'art de contrôler des réseaux sociaux qui fondent la convoitise des hommes politiques. Les courtiers peuvent devenir ministre, député ou recruté comme poulain prometteur d'un parti au pouvoir. Ils s'installent ainsi dans une nouvelle identité professionnelle. Initialement bénévole puis progressivement professionnelle au détriment de la profession d'origine qu'ils n'ont plus le temps ni l'envie d'exercer. Ils se découvrent de nouvelles ressources, de nouvelles compétences, de nouveaux desseins. Ils deviennent vraiment courtiers du développement. Ils le deviennent par la compétence propre qu'ils ont su développer. Mais leur situation est précaire, car ne reposant sur aucune position institutionnelle acquise. Ils doivent sans cesse gérer les ressources qu'ils ont acquises et les faire fructifier. Comme le big man, ils mettent en œuvre des ressources de toute nature, selon la logique des situations, les unes au moyen des autres ${ }^{51}$. Ainsi l'obtention des financements développe leur clientèle et partant leurs réseaux de relations, ce qui accroît leurs possibilités à trouver de nouveaux financements et à conforter ainsi toujours davantage leurs situations. C'est donc à juste titre que J. Boissevain a assimilé le courtier à un entrepreneur ${ }^{52}$. Malgré cette précarité qui est intrinsèque à toute entreprise, on peut dire qu'ils préfigurent certaines transformations sociales provoquées par les politiques de développement dans les pays du tiers monde. Les sociétés se transforment aussi à travers la production de nouveaux acteurs nés de l'État, mais qui s'en éloignent à mesure que leur nouvelle identité s'ancre dans l'espace social.

\section{BIBLIOGRAPHIE}

1) Sur le courtage

Bailey F, 1969. Stratagems and spoil. A social anthropology of politics, Londres : Basil Blackwell (trad. fr. 1971 : Les règles du jeu politique).

Balandier G. 1969. "Les relations de dépendance". Cahiers d'études africaines, IX (35) : 345-349.

Bierschenk T. 1995. "Rituels politiques et construction de l'identité ethnique chez les peuls au Bénin". Cahiers des sciences humaines, 31 (2) : 457-484.

Bierschenk T. \& Olivier de Sardan J.-P. 1993. "Les courtiers locaux de développement". Bulletin de L'APAD 5 : 71-76.

Boissevain J. 1974. Friends of friends. Networks, manipulators and coalition, Oxford : Basil Blackwell.

Blundo G. 1995. "Les courtiers du développement en milieu rural sénégalais", Cahiers d'Etudes

Africaines XXXV-1 (137) : 73-99. 
Cohen A.P. \& Comaroff J.L. 1976. "The management of meaning : on the phenomenology of political transactions". B. Kapferer (sous la dir.) Transaction and meaning, ISHI, Philadelphia : 87-107.

Eisenstadt S. \& Lemarchand R. 1981. Political clientelism, patronage and development. Beverly Hills. Sage.

Geertz C. 1960. "The changing role of the cultural broker : Thejavanese Kijaji". Comparative Studies in Society and History.

Gluckman M. 1949. "The village headman in British central Africa", Africa, 19 (2) : 89-106.

Hill P. 1966. "Landlords and Brokers : a west african trading system". Cahiers d'études africaines, $n^{\circ} 23: 349-366$

Kuper A. 1970. "Gluckman's village headman", American Anthropologist, n72 : 355-358.

Jacob J.P.\& Lavigne Delville P. (eds) 1994. Les associations paysannes en Afrique. Organisation et dynamiques, Paris : APAO-Karthala-IUEO.

Laurent P.J. 1993. "Un «mâle» nécessaire pour le programme de la fédération Wend-Yam", Bulletin de l'APAD, $\mathrm{n}^{\circ} 6: 19-24$

Long N. 1975. "Structural dependancy, mode of production and economic brokerage in rural Peru", in Oxaal I., Bamett T. et Booth D. (eds), Beyond the sociology of development : economy and society in Latin America and Africa. London : Routlege and Kegan Paul.

Long N. 1992. "Du Paradigme perdu au paradigme retrouvé ? Pour une sociologie du développement orientée vers les acteurs", Bulletin de l'APAD, $7:$ 11-34.

Long N. (ed) 1989. Encounters at the interface. A perspective on social discontinuities in rural development, Wageningen. Agricultural University.

Long N. \& Long A. (eds) 1992. Batllefields of knowledge. The interlocking of theory and practice in social research and development, Londres : Routledge.

Lund C. 1995. "Compétition over juridictions and political manoeuvring in Niger", Bulletin de l'APAD $9: 19-29$.

Médard J.F. 1992. "Le «big man» en Afrique. Esquisse d'une analyse du politicien entrepreneur", L'Année sociologique, $42: 167.192$.

Mendras H. 1976. Sociétés paysannes. Eléments pour une théorie de la paysannerie. Paris. Armand Colin (coll. U).

Olivier de Sardan J.P. 1995. Anthropologie et développement. Essai en socio-anthropologie du changement social, Paris : APAD-Karthala.

Robinson P. 1989. "Traditional clientage and political change in Hausa community". in Robinson et Skinner (eds), Resiliency in Africa, Washington. Harward UP : 105-128.

Sahlins M. 1963. "Poor man, Big man, rich Man, chief. Political types in Melanesia". Comparative studies. in Society and History.

2) sur les Touareg

Association Timidria, 1995. Rapport évaluatif du 1er séminaire, tenu à Tahoua du 26 au 28 octobre 1994, Niamey.

Association Timidria, 1995. Rapport évaluatif du forum de réconciliation national, tenu à Tahoua du 28 au 30 septembre 1995. Niamey. 
Association SCOA pour la recherche scientifique en Afrique Noire. Actes des colloques (1er, 2éme et 3ème ).

Bernus E. 1976. Touareg nigériens, Unité culturelle et diversité régionale d'un peuple pasteur. Paris, ORSTOM.

Bernus E. \& S. 1975. "L'évolution de la condition servile chez les touareg sahéliens", in C. Meillassoux (ed.), L'esclavage en Afrique précoloniale, Paris, Maspéro : 27-47.

Bonte P. 1976. "Structure de classe et structures sociales chez les Kel Géres", Romm, n²1 : 141-162.

Boubou H., 1967. Recherches sur l'histoire des touareg sahariens et soudanais, Paris, Présence africaine.

Bourgeot A. 1995. Les sociétés touarègues. Nomadismes, identités, résistances. Paris, Karthala.

Bourgeot A. 1972. "Idéologies et appellations ethniques : l'exemple touareg. Analyse des catégories sociales", Cahiers d'études africaines, ${ }^{\circ} 48$, vol.XII : 533-554.

Claudot-Hawad H. 1993. Les touareg. Portraits en fragments. Paris, Edisud.

Clauzel J. "Les hiérarchies sociales en pays touareg". Travaux de l'institut de recherches sahariennes. T. XXI, 1er semestre : 120-162.

Clauzel J. "L'administration coloniale et les sociétés nomades de l'ancienne Afrique occidentale française", Politique africaine, $\mathrm{n}^{\circ} 46$ : 99-116.

Olivier de Sardan J.P. 1976, Quand nos pères étaient captifs, Paris, Nubia.

Olivier de Sardan J.P. 1982. Concepts et conceptions songhay-zarma. Histoire, culture, sociétés. Paris, Nubia.

Robert D \& Bankoula A.M., 1993. Syndicats et associations au Niger, Niamey.

Salifou A., 1993. La question touarègue au Niger, Paris, Karthala.

Schlechten Rauber M. 1995. "« Timidria », une dynamique inédite au Niger", in Sottas B et Roost Vischer (eds), L'Afrique part tous les matins, Berne, Peter Lang.

Spittler G. 1993. Les touareg face aux sécheresses et aux famines. Les Kel Ewey de l'Aïr (Niger). Paris, Karthala.

\section{NOTES}

1.Bierschenk T. et Olivier de Sardan J.P., 1993, Les courtiers locaux de développement. Sélections d'articles, EHESS-SHADYC, Marseille.

2.Ici nous pensons à plusieurs cas : la figure de Dillali, telle décrite par Hill. Dillali en Haoussa ou Dilan en zarma veut dire intermédiaire. Il est non seulement utilisé dans l'espace marchand, mais dans beaucoup d'autres domaines de la vie sociale. Hill P. Landlords and brokers. A West african trading system, Cahiers d'études africaines, numéro 23, 1966, pp. 359-366.

3.La raison de ce choix doit beaucoup au hasard, mais aussi l'amitié que je porte à certains membres de cette association que j'ai découverte par leur entremise. Je voudrai ici les remercier de m'avoir permis de travailler avec eux durant tous ces mois. 4.Barbedette L., Ouédraogo J. op.cit p. 36. Les chiffres rapportés par ces auteurs sont impressionnants. Il m'a été impossible de les vérifier sur le terrain. La lecture de 
certains rapports de mission effectuées par les membres du bureau de l'association accrédite l'idée d'une forte implantation rurale de l'association.

5.Schlechten Rauber, "Timidria", une dynamique inédite au Niger, in Sottas B. et Roost Vischer (eds), L 'Afrique part tous les matins. Berne, Peter Lang, 1995. Au moment où elle écrivait cet article. Mme Schlechten Rauber était en poste au bureau de coordination de la DDA à Niamey.

6.Barbedette L., Ouédraogo J. Place des organisations du monde rural dans l'éclosion associative au Niger, Note de mission, octobre 1993, p. 36. Ce document a été réalisé sous l'égide de la coopération suisse et l'association internationale SIX S.

7.Cf. infra.

8.Association Timidria, 1995, Rapport évaluatif du 1er séminaire tenu à Tahoua du 26 au 28 octobre 1994, Niamey.

9.Long N. "Du paradigme perdu au paradigme...retrouvé ? Pour une sociologie du développement orientée vers les acteurs", Bulletin de l'APAD n, juillet 1994, p. 15.

10.Barbedette L., Ouédraogo J. op.cit. On se reportera aussi avec intérêt à : Robert D., Bankoula A.M :, Syndicats et associations au Niger, Niamey, mars 1993. Ce document recense la liste complète des associations et syndicats du Niger, avec la date de leur création et le nom de leur principal responsable.

11.L'étude relève qu'en 25 ans, 54 associations de ce type voient le jour. Mais peu d'entre elles continue aujourd'hui de se créer. Alors qu'elles occupaient $75 \%$ du paysage associatif en 1985, en 1993, les associations culturelles et sportives ne représentent plus que $20,5 \%$ de ce paysage, et les autres associations de cette première génération, $14 \%$.

12.En 1993, les ONG représentaient une proportion de $39 \%$ des associations officiellement reconnues au Niger.

13.A.P. Cohen et J.L. Comaroff, The management of meaning: On the phenomenology of political transactions, in B. Kapferer (sous la dir.) Transaction and meaning, ISHI, Philadelphia, 1976, p.88

14.L'expression est L. Barbedette et de J. Ouédraogo. op.cit. p. 36. "Intellectuels" doit être entendu ici au sens large, incluant tous ceux qui sont allés à l'école. Ici, l'intellectuel se définit surtout par rapport au rural resté à la marge du système éducatif de l'Etat.

15.Selon Bourgeot, les oppositions touareg blancs/touareg noirs datent de la l'époque coloniale. Selon le même auteur, elles sont le produit d'une conception raciste en accord avec l'idéologie de l'époque. La catégorie "Touareg blanc" représente les "suzerains" et les "plébéiens vassaux", tandis que celle des "touareg noirs" recouvre l'ensemble des asservis et des tributaires. cf. Bourgeot A., 1995, Les sociétés touarègues. Nomadisme, identité, résistances, Paris, Karthala, p. 29 et suite).

16.Document-cadre de la communauté touareg de souche noire, Multigraphié, Niamey, non daté.

17.ibid. p.1, notamment le préambule.

18. C'est moi qui traduit du Haoussa.

19.Cf. Annexe 2 du dossier de la requête introduite par Timidria à l'USAID pour obtenir le financement du séminaire de Tahoua. Cet annexe est relative à la description du projet.

20.Les objectifs de l'association Timidria peuvent être repérés au titre II des statuts de l'association. 
21.Ici on se réfèrera à la lettre ouverte adressée aux autorités de la troisième république nigérienne par les "touareg de peau noire". Cf. Le Démocrate, du Lundi 13 juin 1994 aux pages 2 et 7 ; On se réfèrera aussi à un document multigraphié intitulé "Document-cadre de la communauté touareg de souche noire" dont le porte-parole mentionné est justement le Président de Timidria.

22.Cf. Document-cadre, op.cit.

23.E. Bemus, Touareg nigériens, Unité culturelle et diversité régionale d'un peuple pasteur, Paris. ORSTOM.

24.La distinction de Bemus semble s'appuyer beaucoup sur celle élaborée par Clauzel en 1962. cf. Les hiérarchies sociales en pays touareg, Travaux de l'institut de recherches sahariennes t. XXI, 1 er semestre, p. 120-162.

25.Boubou Hama, in Actes du séminaire de l'Association SCOA, Niamey, du 14 au 21 janvier 198 1, p. 155- 158. Pour Boubou Hama, le nom bella ne désigne pas que le noir. Il s'est étendu par la suite à certains vassaux qui ont fini par se fondre dans la masse des bella. Ces derniers ne sont donc pas tous noirs. Il y en aurait, selon lui, qui comptent des types très clairs avec des cheveux lisses et longs.

26.J.P. Olivier de Sardan, Concepts et conceptions songhay-zarma. Histoire, culture. société. Paris, Nubia, 1982, p. 65.

27.E. Bemus, op.cit., p. 91.

28.lbid.

29.L'origine des Iklan est difficile à établir. Pour certains, ils seraient les descendants $\mathrm{du}$ fond de population noire autochtone sahélo-saharienne, auquel sont venus s'adjoindre les soudanais razziés. Cette hypothèse se réfère aux touareg du Sahara. Dans l'ouest du Niger, on les affilie directement aux sorkos, et ce à une époque bien antérieure à celle que l'historiographie nous donne sur l'arrivée des touareg dans la région. Pour cette hypothèse voir, Boubou Hama, op.cit., p. 156, J.P. Olivier de Sardan, op.cit., p. 66. Cependant, ces origines ne semblent pas influer sur l'identité des Iklans, puisque l'enquête montre qu'ils ont presque tous perdu tout souvenir de leur origine. J.P. Olivier de Sardan, ibid.

30.On sait bien que beaucoup de touareg Illelan sont de peau noire, et ceux quelle que soit la strate considérée. Tout comme Boubou Hama rapporte que les Bella ne sont pas tous noirs. J.P. Olivier de Sardan, ibid.

31.Cependant, il faut se garder de penser que les tous les touareg dits de souche noire vivent dans des conditions de servitude Au sein même de l'association certain militant affirme clairement qu'ils ne sont pas des Iklan. Par ailleurs, lors du séminaire de Tahoua, sur lequel nous reviendrons, l'un des conférenciers, Docteur El Back, qui de par son appartenance ethnique, est touareg du Damergu. affirme que dans leur régions, ils ne connaissent cette dichotomie, qui semble selon lui être propre à l'Azawagh. Par ailleurs Boubou Hama rapporte des éléments assez intéressants pour éclairer cette question.

32.Il est peut-être utile de nuancer cette affirmation. L'étude de Spittler sur les Kel Ewey de l'Aïr ne parle à aucun moment des Iklan et encore moins de "touareg de souche noire". Cf. Spittler G. 1993, Les Touaregs face aux sécheresse et aux famines. Les Kel Ewey de l'Air, Paris, Karthala.

33.Par exemple, P. Bonte, L'organisation économique des touareg Kel Gress, in R. Cresswell, Eléments d'ethnologie, Vol. 1, p. 182 et suite consacrés aux rapports sociaux de production. 
34.Il vient à l'esprit ici l'ouvrage Gerd Spittler, Les touareg face aux sécheresses et aux famines. Les Kel Ewey de l'Aïr (Niger), Karthala, 1993. Le sous-titre de l'ouvrage correspond mieux à la réalité du terrain étudié, ce qui exclut d'emblée le groupe auquel nous faisons référence.

35.Par exemple, sur financement du Centre de Coopération Technique et Rural (CTA) basé au Pays-Bas, le Président de Timidria a effectué au Burkina Faso une mission d'études en agroforesterie. Cette mission a été pour lui l'occasion de rencontrer des ressortissants du Mali, du Bénin, de la Mauritanie, du Sénégal, du Tchad, du Burkina Faso.

36.Pierre-Joseph Laurent, Un mâle nécessaire pour le programme de la fédération Wend-Yam. Bulletin de l'APAD, nº, 1993, pp. 19-24. Cette expression me paraît bien exprimer le rôle des courtiers qui m'ont intéressé mais à condition de comprendre le projet dans son acception la plus large, en ne le limitant pas au projet de développement. Par ailleurs, la rente à négocier que je comprends comme une forme de rétribution doit être liée à des succès dans les entreprises initiées par les courtiers, avec ce que cela implique en termes d'incertitudes, à condition aussi que cette rétribution ne soit pas limitée à des considérations d'ordre exclusivement matériel.

37.0p.cit., p. 90.

38.Norman Long. Encounters at the interface. A perspective on social discontinuities in rural development. Agricultural University, 1989, Wageningen, p. 5.

39.Par exemple quand le PNUD a adopté son approche-programme comme méthode devant lui permettre d'identifier, de concevoir et de gérer ses projets, il a organisé un séminaire qui a réuni les hauts fonctionnaires concernés par sa coopération pour les familiariser avec ses nouveaux concepts et démarches.

40.Ceux-là connaissent le milieu des bailleurs. Ils connaissent les interstices du monde du financement international. Ils y ont souvent développé des relations personnelles. Souvent même, ils sont engagés dans la voie associative à l'instigation de ces derniers, avec des promesses fermes de financement.

41.Je pense ici à des associations comme Démocratie-Liberté-développement (DLD), à l'alliance nigérienne pour les droits et l'homme (ANDDH), pour ne citer les plus en vue. Les associations des droits de l'homme ont été très présentes sur la scène politique lors des élections législatives anticipées de janvier 1995. Timidria ne figurait pas parmi elle, alors même que l'un des léitmotiv de sa lutte l'en prédisposait.

42.Notre rencontre, en présence du bureau de la section et des membres de la coopérative, le dimanche 26 février 1995 à Maradi.

43.Il ne m'a pas semblé que les dirigeants de Timidria avaient des références précises sur l'établissement à Maradi des populations qu'elle cherche à mobiliser. Les informations qu'ils m'ont données sont assez générales. Si elles concordent pour une partie de la population concernée, il y a des trajectoires autres. Par celle de Alk qui m'a dit qu'il était venu à Maradi avec un projet qui l'a recruté à Tahoua. Il a suivi le projet un peu partout avant de se retrouver à Maradi où il travaille à l'hôpital de la ville en qualité de gardien. El hadj, pour sa part est marabout. Il semble s'être installé à Maradi. en raison des potentialités que présente la ville pour ses activités.

44.Entretien du 4 novembre 1994. Je transcris exactement le discours qu'il m'a tenu.

45.Au Niger, c'est le service civique qui a remplacé le service militaire.

46.Le cours normal de Tahoua formait des instituteurs-adjoints.

47.G. Blundo, Les courtiers du développement en milieu rural sénégalais, Cahiers d'études africaines, n¹37, 1995, p. 73-99. 
48.Ibid., pp. 79-86.

49.P.T. Robinson, Traditional clientage and political change in a hausa community, in Robinson and Skinner (eds), Resiliency in Africa, Washington :Harward UP, pp. 105-128. 50.0p. cit. pp. 90-95. Le courtier décrit par les auteurs utilisent ses ressources pour permettre à une communauté locale d'accéder au ressources du gouvernement. 51.J.F. Médard, Le "Big man" en Afrique : Esquisse d'analyse du politicien entrepreneur. L'année sociologique, $\mathrm{n}^{\circ} 42,1992, \mathrm{p} .168$.

52.J. Boissevain, Friends of friends, networks, manipulators and coalitions. Oxford, Basil Blackwell, 2ème édition, 1978, p. 147.

\section{AUTEUR}

MAHAMAN TIDJANI ALOU

Ministère des Affaires Étrangères et de la Coopération, BP 396, Niamey (Niger) Tél. $+227 / 73.52 .31$. 